\title{
Haplotype-based case-control study of calpain (CAPN2) gene and hypertensive disorders of pregnancy
}

\author{
T. Nakayama ${ }^{1}$, T. Nakayama ${ }^{2,3}$, K. Shinya ${ }^{1}$, E. Shikata ${ }^{2}$, K. Kawana ${ }^{1}$ \\ and T. Yamamoto ${ }^{1}$ \\ ${ }^{1}$ Department of Obstetrics and Gynecology, Nihon University School of \\ Medicine, Tokyo, Japan \\ ${ }^{2}$ Division of Laboratory Medicine, Department of Pathology and \\ Microbiology, Nihon University School of Medicine \\ ${ }^{3}$ Division of Companion Diagnostics, Department of Pathology of \\ Microbiology, Nihon University School of Medicine \\ Corresponding author: T. Nakayama \\ E-mail: nakayama.tomohiro@nihon-u.ac.jp
}

Genet. Mol. Res. 20 (1): gmr18639

Received May 13, 2020

Accepted February 20, 2021

Published March 31, 2021

DOI http://dx.doi.org/10.4238/gmr18639

\begin{abstract}
Various theories have been developed regarding the pathology and etiology of hypertensive disorders of pregnancy (HDP). T he impaired placenta secretes antiangiogenic factors into maternal blood, resulting in the development of pregnancy-induced hypertension $(\mathrm{PIH})$. Both $\mu$-calpain and $\mathrm{m}$-calpain are proteins known to be involved in placentation, with the catalytic subunits encoded by the CAPN1 and CAPN2 genes, respectively. The purpose of this study was to find disease susceptibility genes for PIH by conducting an association analysis for HDP using specific gene markers comprising individual single nucleotide variants (SNVs) and haplotypes within the CAPN2 gene. We selected five SNVs in the human CAPN2 gene and performed an association study with 95 HDP patients and 177 age-matched non-HDP subjects. In the analyses conducted for the CAPN2 SNVs, the recessive model of rs1153968 differed significantly between the gestational hypertension (GH, a category of HDP) and control groups . However, rs1 153968 deviated from Hardy-Weinberg equilibrium and was deemed unlikely
\end{abstract}


to be a significant factor. No significant differences were seen in any of the other SNVs analyzed. However, association analyses involving the rs1892077-rs98041140-rs17599-rs1153954 haplotype in the CAPN2 gene haplotypes revealed that G-A-A-T was found at a significantly lower frequency, and G-A-C-T and G-G-A-A at significantly greater frequencies, in patients with $\mathrm{PIH}$. These findings may be useful in preventing PIH onset, as well as for the early discovery and treatment of patients who are carriers of the haplotype likely to be associated with this condition

Key words: Hypertensive disorders of pregnancy; Calpain; Variant; Haplotype; Association study

\section{INTRODUCTION}

Hypertensive disorders of pregnancy (HDP) have been suggested to result from a variety of causes. Since HDP is thought to be induced by a two-step mechanism, the pathology of HDP likely originates in a background of placental dysfunction (Chen et al., 2017). Many causes of HDP have been reported, and HDP is believed to be a multifactorial genetic disorder (Guo et al., 2016). The main mechanisms involved in HDP are believed to include damage to endothelial cells, thrombotic tendencies, placenta dysfunctions, and oxygen deficiencies (Gu et al., 2017).

Calpains are Ca-dependent cysteine proteases that are primarily present in the cytoplasm. Calpains are composed of 15 kinds of catalytic subunits and two kinds of regulatory subunits, which together form heterodimers, consisting of one catalytic subunit and one regulatory subunit. Catalytic subunits can consist of any of six tissue-specific types or of any of nine non-tissue-specific types. Among calpains with a nonspecific catalytic subunit, two types ( $\mu$-calpain and m-calpain) have been the subjects of advanced functional analysis (Goll et al., 2003). Notably, $\mu$-calpain and m-calpain share the same regulatory subunit, CAPNS1, but differ in their catalytic subunit, with $\mu$-calpain employing CAPN1, and m-calpain employing CAPN2 (Guo et al., 2016).

Calpastatin (the product of the CAST gene) is an intracellular protein that serves as a calpain inhibitor by selectively inhibiting that protein. The calpain-calpastatin system has been implicated in many disease states, for example Alzheimer's, muscular dystrophy, cataracts, and lissencephaly (Saido et al., 1994; Zatz and Starling, 2005; Yanada et al., 2009).

In an embryological study of calpain, CAPNS1 knockout mice exhibited embryonic lethality around embryonic day 10.5, suggesting that $\mu$ - and m-calpain are important for fetal growth. CAPN1 knockout mice exhibited normal growth but revealed abnormalities in the coagulation system, including decreased platelet aggregation and clot retraction (Azam et al., 2001). CAPN2 knockout mice exhibited embryonic lethality around embryonic day 2.5 , as a result of placental hypoplasia (Dutt et al., 2006). Conditional expression of CAPN2 in the placenta counteracted this lethality (Takano et al., 2011).

Together, these studies indicate that m-calpain is essential for fetal development, especially in placentation. Comparison of CAPNS1 knockout mice (which do not express $\mu$ or m-calpain) and $C A P N 2$ knockout mice (which do not express m-calpain) demonstrate a 
difference in the time taken to reach embryonic lethality, implying that $\mu$-calpain also is involved in placentation.

We conducted a haplotype-based case-control study to assess the association between HDP and single-nucleotide variants (SNVs) in the human CAPN2 gene.

\section{MATERIAL AND METHODS}

\section{Subjects}

HDP is defined as hypertension, with or without proteinuria, occurring after the 20th week of gestation but resolving by the 12th week postpartum. HDP is classified into four categories: i) preeclampsia (PE), ii) gestational hypertension $(\mathrm{GH})$, iii) superimposed $\mathrm{PE}$, and iv) eclampsia. $\mathrm{PE}$ is considered hypertension with proteinuria occurring after the 20th week of gestation but resolving by the 12th week postpartum. GH is hypertension without proteinuria occurring after the 20th week of gestation but resolving by the 12th week postpartum. These criteria are fundamental characteristics of HDP as defined by the International Society for the Study of Hypertension in Pregnancy (ISSHP) (Brown et al., 2001).

The cases examined in our study were selected from a pool of 167 patients with HDP and 266 control patients. All invididuals had been admitted to our hospital from 2006 to 2015. We excluded any cases for which a genotype could not be defined for all of the SNVs evaluated in this study (Figure 1). As a result, the cases examined in the this study included $81 \mathrm{PE}, 14 \mathrm{GH}$, and 177 controls.

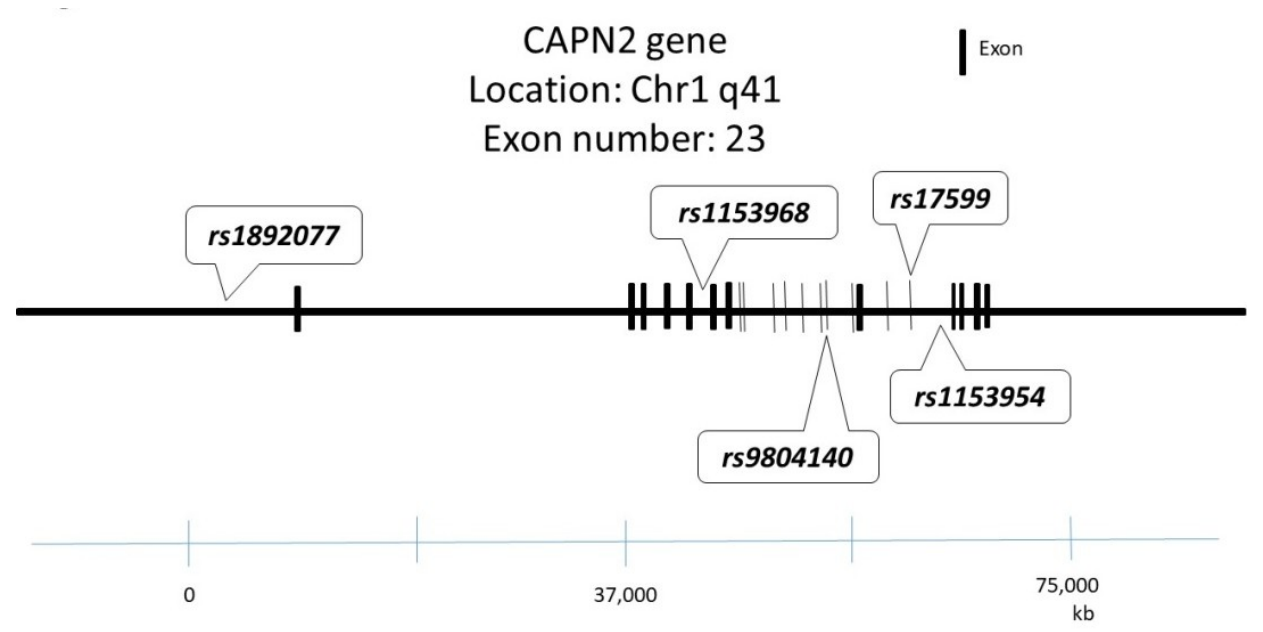

Figure 1. Organization of the CAPN2 gene and locations of the single nucleotide variants

Informed consent was obtained from each participant. This study was approved by the Human Studies Committee of Nihon University, School of Medicine (Approval No. 168-1). 


\section{Genotyping}

Based on the information obtained from the National Center for Biotechnology Information (NCBI) database web site, we chose SNVs with a minor allele frequency (MAF) of $>5 \%$. We selected five SNVs in the human CAPN2 gene. Blood samples were collected from all patients, and genomic DNA was isolated from peripheral blood using standard methods. Genotypes were determined using Assays-on-Demand kits (Applied Biosystems, Branchburg, NJ) and TaqMan PCR as the standard methods (Shinya et al., 2018).

\section{Statistical analysis}

For statistical analysis, significant differences between the groups were assessed using the Mann-Whitney U test. Analysis of the correlation was examined by the $\chi^{2}$ test. Tests were performed using GraphPad Prism 6 (GraphPad Software, Inc., San Diego, CA, USA). Differences with $P$ values of $<0.05$ were considered significant.

The linkage disequilibrium (LD) analysis and haplotype-based case-control study were conducted using SNPAlyze (Windows version) Ver. 3.23 (Dynacom, Chiba, Japan). Haplotypes observed at frequencies of less than 0.01 were excluded. Differences with $\mathrm{P}$ values of $<0.05$ were considered significant (Tamura et al., 2008).

\section{RESULTS}

For our haplotype-based case-control study, we excluded cases in which the genotypes of all of the SNVs could not be determined. Following this exclusion condition, the study enrolled 177 cases in the control group and 95 cases in the HDP group; the HDP group included $81 \mathrm{PE}$ cases and $14 \mathrm{GH}$ cases.

The characteristics of the participants are summarized in Table 1. There were no significant differences in the age or the frequency of primigravidas between the control and HDP groups (control vs. HDP, vs. PE, vs. GH). Body mass index (BMI) immediately before delivery, systolic blood pressure (SBP), and diastolic blood pressure (DBP) were significantly higher in the HDP group than in the non-HDP group. The gestational age at delivery (weeks), birth weight of neonates, and Apgar score were significantly lower in the HDP group than in the non-HDP group. BMI before pregnancy was significantly higher in the HDP group and in the PE group than in the non-HDP group. The gain of body weight during the pregnancy period was significantly higher in the PE group than in the non-HDP group. The frequency of family history of hypertension was significantly higher in the GH group than in the non-HDP group.

The nucleotide sequence and minor allele frequency (MAF) of each SNV are provided in Table 2. The results of the association analysis for the five selected SNVs are provided in Supplementary 1. The MAFs of the recessive allele of rs1153968 differed significantly between the GH and non-HDP groups $(\mathrm{P}=0.012)$. However, the frequency of this SNV did not satisfy Hardy-Weinberg equilibrium, indicating that this result was not meaningful. Significant differences were not apparent for the other SNVs. 
Table 1. Characteristics of study participants (CAPN2).

\begin{tabular}{|c|c|c|c|c|c|c|c|}
\hline & $\begin{array}{l}\text { Control } \\
(n=177)\end{array}$ & $\begin{array}{l}\text { HDP } \\
(n=95)\end{array}$ & $\begin{array}{l}\text { P values } \\
\text { compared } \\
\text { with control }\end{array}$ & $\begin{array}{l}P E \\
(n=81)\end{array}$ & $\begin{array}{l}\text { P values } \\
\text { compared } \\
\text { with } \\
\text { control } \\
\end{array}$ & $\begin{array}{l}\text { GH } \\
(n=14)\end{array}$ & $\begin{array}{l}\text { P values } \\
\text { compared } \\
\text { with } \\
\text { control }\end{array}$ \\
\hline Age (years) & $32.0 \pm 7.6$ & $31.2 \pm 5.1$ & 0.985 & $31.2 \pm 4.9$ & 0.128 & $31.5 \pm 5.8$ & 0.942 \\
\hline $\mathrm{DBP}(\mathrm{mmHg})$ & $73.2 \pm 10.6$ & $98.0 \pm 19.8$ & $<0.001$ & $100.2 \pm 20.0$ & $<0.001^{*}$ & $88.4 \pm 15.4$ & $<0.001 *$ \\
\hline $\begin{array}{l}\text { BMI before pregnancy } \\
\left(\mathrm{kg} / \mathrm{m}^{2}\right)\end{array}$ & $20.9 \pm 3.1$ & $22.9 \pm 5.2$ & $0.007^{*}$ & $23.1 \pm 5.4$ & $0.004 *$ & $22.1 \pm 4.5$ & 0.670 \\
\hline $\begin{array}{l}\text { Body weight gain during } \\
\text { pregnancy }(\mathrm{kg})\end{array}$ & $10.3 \pm 3.8$ & $8.9 \pm 5.9$ & 0.051 & $8.3 \pm 6.0$ & 0.015 & $11.4 \pm 4.5$ & 0.63 \\
\hline $\begin{array}{l}\text { Gestational age at delivery } \\
\text { (weeks) }\end{array}$ & $38.6 \pm 1.6$ & $35.9 \pm 3.5$ & $<0.001^{*}$ & $35.4 \pm 3.5$ & $<0.001^{*}$ & $36.5 \pm 3.1$ & $0.011^{*}$ \\
\hline Birth weight of neonates $(\mathrm{g})$ & $3055.0 \pm 474.1$ & $2247.9 \pm 828.2$ & $<0.001^{*}$ & $2155.7 \pm 821.3$ & $<0.001^{*}$ & $2623.4 \pm 746.4$ & $0.048^{*}$ \\
\hline Apgar score & $8.7 \pm 0.6$ & $7.5 \pm 2.2$ & $<0.001^{*}$ & $7.4 \pm 2.3$ & $<0.001^{*}$ & $7.8 \pm 1.3$ & $0.033^{*}$ \\
\hline $\begin{array}{l}\text { Frequency of primigravidas } \\
(\%)\end{array}$ & 61.0 & 54.1 & 0.464 & 55.0 & 0.295 & 50.0 & 0.328 \\
\hline
\end{tabular}

HDP, hypertensive disorders of pregnancy; PE, preeclampsia; GH, gestational hypertension. SBP, systolic blood pressure; DBP, diastolic blood pressure; BMI, body mass index; HT, hypertension. Continuous variables are expressed as mean \pm standard deviation. Categ orical variable are expressed as percentage. The $\mathrm{P}$ values for the continuous variables were calculated using the Mann-Whitney U test. *: $\mathrm{P}$ value $<0.05$

Table 2. SNVs in CAPN2 gene.

rs1892077 MAF: G=0.30 Location: 5'-upstream

ACTCACTCCAGGAAGGTTTTCCACC[A/G]TGAAGTGTTTGTCTATGTCCTTGTG

rs1153968 MAF: $A=0.46 \quad$ Location: Intron 5

AGCCCTGACTTGAACTCAACCTCTT[A/C]CTCCTCCCTTTTATCTAATCCTGCA

rs9804140 MAF: G=0.05 Location: Exon 14

CTCCGGGAGGTGCTCAACCGCTTCA[A/G]GCTGCCGCCAGGAGAGTACATTCTC

rs17599 MAF: $\mathbf{C}=\mathbf{0 . 2 0}$ Location: Exon 18

ATTTTGTCTTTTAGGCCAAGATATC[A/C]AGTCAGATGGCTTCAGCATCGAGAC

rs1153954 MAF: A=0.30 Location: Intron 18

GAAAGACCTGCTGGCTCCCTGCCGA[A/T]TCTGGTTGCTGTTTTGATCTAGGGG

MAF: minor allele frequency. ${ }^{*}$ The nucleotides in the exonic regions are indicated by italics

Because all r-squared values calculated for the remaining four SNVs (non- rs1153968) were small $\left(\mathrm{r}^{2}<0.5\right)$, we constructed a haplotype-based association study using these four SNVs (Table 3). Specifically, the combination of the four selected SNVs together corresponded to two susceptibility haplotypes (G-A-C-T and G-G-A-A) and one resistance haplotype (G-A-A-T) (Table 4).

Table 3. r-squared values for $C A P N 2$ SNVs.

\begin{tabular}{|c|c|c|c|c|}
\hline SNVs & rs1892077 & rs9804140 & rs17599 & rs1153954 \\
\hline rs1892077 & & 0.007 & 0.035 & 0.012 \\
\hline rs9804140 & & & 0.005 & 0.016 \\
\hline $\begin{array}{l}\text { rs17599 } \\
\text { rs } 1153954\end{array}$ & & & & 0.001 \\
\hline
\end{tabular}

Gray-shaded cells: r-squared $<0.5$

Genetics and Molecular Research 20 (1): gmr18639 


\begin{tabular}{|c|c|c|c|c|c|c|c|}
\hline \multicolumn{8}{|c|}{$\begin{array}{l}\text { overall P value } \\
0.0001\end{array}$} \\
\hline Haplotype & $\begin{array}{l}\text { rs1892077 } \\
\text { A/G } \\
\end{array}$ & $\begin{array}{l}\text { rs9804140 } \\
\mathrm{A} / \mathrm{G}\end{array}$ & $\begin{array}{l}\text { rs17599 } \\
\mathrm{A} / \mathrm{C}\end{array}$ & $\begin{array}{l}\text { rs1153954 } \\
\text { A/T } \\
\end{array}$ & Control & PIH & P value \\
\hline H1 & $\mathrm{A}$ & $\mathrm{A}$ & $\mathrm{A}$ & $\mathrm{T}$ & 0.378 & 0.406 & 0.561 \\
\hline $\mathrm{H} 2$ & G & A & A & $\mathrm{T}$ & 0.064 & 0.000 & $<0.001^{*}$ \\
\hline $\mathrm{H} 3$ & A & G & A & $\mathrm{T}$ & 0.069 & 0.035 & 0.073 \\
\hline H4 & G & G & A & $\mathrm{T}$ & 0.031 & 0.025 & 0.737 \\
\hline H5 & A & A & C & $\mathrm{T}$ & 0.197 & 0.188 & 0.772 \\
\hline H6 & G & A & $\mathrm{C}$ & $\mathrm{T}$ & 0.000 & 0.019 & $0.007^{*}$ \\
\hline H7 & A & G & C & $\mathrm{T}$ & 0.032 & 0.052 & 0.224 \\
\hline H8 & A & A & A & A & 0.066 & 0.086 & 0.429 \\
\hline H9 & A & G & A & A & 0.051 & 0.077 & 0.297 \\
\hline H10 & G & G & A & A & 0.000 & 0.020 & $0.007^{*}$ \\
\hline H11 & A & A & $\mathrm{C}$ & A & 0.078 & 0.041 & 0.114 \\
\hline $\mathrm{H} 12$ & G & A & $\mathrm{C}$ & A & 0.000 & 0.010 & 0.055 \\
\hline H13 & A & $\mathrm{G}$ & $\mathrm{C}$ & A & 0.034 & 0.041 & 0.647 \\
\hline
\end{tabular}

\section{DISCUSSION}

In a comparison of the clinical characteristics between the HDP and control groups patients with HDP exhibited significantly higher SDP, DBP, pre-pregnancy BMI, postpregnancy BMI, amount of weight gain, and frequency of a family history of hypertension; and significantly lower gestational age at delivery (weeks), birthweight, and Apgar score. Indeed, elevations in SDP and DBP are defining characteristics of HDP. Moreover, delivery itself becomes a mode of treatment when HDP is exacerbated, even if this "treatment" results in preterm delivery. Consequently, gestational age at delivery is expected to be lower in patients with HDP, leading to lower birthweight and lower Apgar score in the resulting babies. These parameters are thought to be outcomes specific to HDP. Family history of hypertension, pre-pregnancy obsesity, and gestational weight gain are known risk factors for HDP. Thus, although some clinical background variables exhibited significant differences, all the variables were specific to the condition of HDP itself and so should be significantly different as long as HDP is the study's target disease. These results are not considered to affect association analyses that use individual SNVs or haplotypes. Given that calcium-related molecules are associated with endothelium-dependent vascular contraction, HDP-related susceptibility genes are worthy of examination in association studies (Nagai et al., 2012; Shinya et al., 2018).

Few previous studies have performed association analyses on the haplotypes of calpain-related genes. Regarding the calpain gene investigated in our study, we identified a single relevant paper that analyzed the CAPN1 gene in birds and cows in the context of meat quality and muscle physiology (Cushman et al., 2015; Shu et al., 2015). For other calpain-related genes, a handful of association analyses reported on the associations of the CAPN10 gene (calpain 10 encodes another calpain subunit) in polycystic ovarian syndrome and in diabetes types 1 and 2 (Xiang et al., 2001; Wang et al., 2002; Wu et al., 2005; Lee et al., 2009). To the best of our knowledge, no previous analyses have examined the association of calpain genes with perinatal conditions such as HDP. 
On the other hand, research on calpain at the protein level continues to progress , and, as described in the Introduction, associations with many conditions and diseases have been suggested. There are many reports of $\mu$-calpain and $\mathrm{m}$-calpain and the involvement of these proteins in the perinatal period. For example, it has been reported that m-calpain expression is elevated in the placenta of mice with Group B streptococcal infection (Equils et al., 2009) and that $\mu$-calpain expression is significantly increased (and m-calpain and calpastatin expression unchanged) in the decidual epithelial cells of patients with recurrent miscarriages (compared to controls) (Kumagai et al., 2008). Based on those reports, the calpain/calpastatin system appears to be involved in HDP.

In particular, since $\mu$-calpain expression is increased in recurrent miscarriage and CAPN1-knockout mice (which do not express $\mu$-calpain) develop normally (Zatz and Starling., 2005), an increase in $\mu$-calpain expression in combination with an increase in CAPN1 gene expression is a risk factor for the development of HDP. We therefore postulated that the haplotype observed with a significantly greater frequency in HDP, based on our present association analysis of CAPN1 haplotypes, may contribute to the upregulation of the CAPN1 gene. On the other hand, previous work did not detect a difference in m-calpain expression between recurrent miscarriage and control groups, although CAPN2-knockout mice that do not express m-calpain develop placental hypoplasia, resulting in embryonic death (Yamada et al., 2009). That researchindicated that a consensus has yet to be reached regarding the association between $\mathrm{m}$-calpain expression and HDP.

In our study, we conducted an association analysis for the calpain-calpastatin system, specifically focusing on $\mu$-calpain, which has been suggested to play a role in placentation. The focus of our study, the CAPN2 gene, encodes the catalytic subunit of mcalpain. A more complete examination of the calpain-calpastatin system would require analysis of the CAPNS1 gene, which encodes a regulatory subunit of both $\mu$-calpain and mcalpain, as well as the $C A S T$ gene, which encodes the calpain inhibitor calpastatin. In fact, the CAPNS1 and CAST genes already have been reported to be involved in mouse placentation (Azam et al., 2001).

We used genetic variants (in the form of SNVs) as gene markers (landmarks). Although no significant differences compared to the control group were seen in the analysis of individual SNVs (Supplementary 1), the haplotype-based association analysis revealed significant differences. There are two SNVs in the CAPN2 gene, rs9804140 and rs17599, that result in missense mutations in the encoded protein; according to the NCBI database, these alleles occur frequently, with MAFs of 0.05 and 0.20 (respectively) in the general population. In our study, association analysis using SNVs did not demonstrate significant differences, indicating that these individual SNVs are not associated with HDP. However, associations were observed for haplotypes (Figure 2), showing the advantages of conducting association analyses using haplotypes . Even when there are no significant differences between the case and control groups in the frequencies of alleles for each individual SNV, a significant difference can exist between the two groups when the analysis utilizes SNV combinations on one chromosome. For example, in our study, both the case and control groups had the same frequency distribution, with four As and two Ts at SNV1, four Ts and two Gs at SNV2, and four Gs and two As at SNV3. However, evaluation of the SNV combination on one chromosome (the haplotype) revealed that the A-T-G haplotype was observed only in the case group, and not at all in the control group. Thus, this haplotype is a 
useful gene marker and is evidence that a link exists to a definitive variant (mutation) related to gene function (as shown by the Xs in Figure 2). In humans, individual SNVs do not exist alone, but rather are clustered together on a given chromosome. Thus, association analyses using haplotypes that compare the combination of SNVs on a given chromosome have a greater detection power than do analyses using individual SNVs, and they may identify gene markers that are of greater use.

In our association analysis, the rs1892077-rs98041140-rs17599-rs1153954 haplotype of the CAPN2 gene (corresponding to G-A-C-T and G-G-A-A sequences) was a useful gene marker of HDP. A single patient in our study population was heterozygous for G-A-C-T and G-G-A-A at the CAPN2 gene. This patient was in the PE group and presented with a blood pressure of $\geq 160 / 90 \mathrm{mmHg}$, meeting the diagnostic criteria for severe HDP. On the other hand, no individuals in the control group possessed multiple haplotypes considered to be gene markers of HDP. Based on these findings, the haplotype thought to comprise gene markers for HDP (as revealed in this study) is not only useful as a gene marker, but also contributes additively or synergistically to the exacerbation of HDP.

\section{Case group}

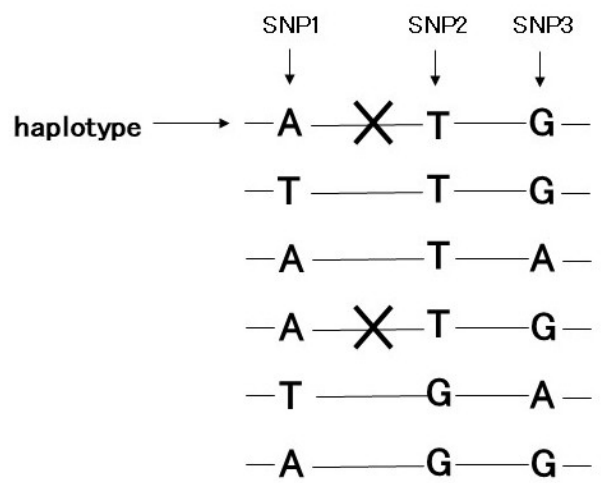

Control group

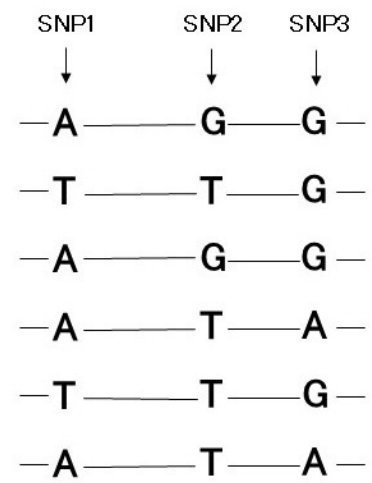

Figure 2. An example showing the advantage of performing haplotype-based association studies. As an example of the advantage of performing haplotype-based association studies, both the case and control groups had the same frequency distribution for SNVs, with 4 As and 2 Ts at SNV1, 4 Ts and 2 Gs at SNV2, and 4 Gs and 2 As at SNV3. However, looking at the SNV combinations on one chromosome (the haplotype), the A-T-G haplotype was observed only in the case group, and not in the control group. Thus, this haplotype is a useful gene marker and suggests that a link exists to a definitive variant (mutation) associated with the cause of disease (as shown by the Xs in the figure).

In our study, we conducted an association analysis of HDP and CAPN2 SNVs and demonstrated, for the first time, that the CAPN2 gene may be a marker for HDP. In the future, if signifcant differences are found in association analyses with the CAPNS1 or CAST genes, these loci might be used together as markers for HDP. Identification of the germline variant in genes associated with HDP may facilitate elucidation of the disease by sequence analysis or other molecular methods (Cho et al., 2009).

\section{ACKNOWLEDGMENTS}

We wish to thank Ms. K. Sugama for her technical assistance. This work was supported by a grant from the Health Sciences Research Institute, Inc. (Yokohama, Japan), 
to the Division of Companion Diagnostics, Department of Pathology and Microbiology, Nihon University School of Medicine (Tokyo, Japan). This work was supported by JSPS KAKENHI Grant Number JP25460703.

\title{
CONFLICTS OF INTEREST
}

\author{
The authors declare no conflict of interest.
}

\section{REFERENCES}

Azam M, Andrabi SS, Sahr KE, Kamath L, et al. (2001). Disruption of the mouse mu-calpain gene reveals an essential role in platelet function. Mol. Cell Biol. 21: 2213-2220.

Brown MA, Lindheimer MD, de Swiet M, Van Assche A, et al. (2001). The classification and diagnosis of the hypertensive disorders of pregnancy: statement from the International Society for the Study of Hypertension in Pregnancy (ISSHP). Hypertens Pregnancy. 20: IX-XIV.

Chen J, Zhong M and Yu YH (2017). Association between interleukin-4 polymorphisms and risk of pre-eclampsia in a population of Chinese pregnant women. Genet. Mol. Res. 16: gmr16029218.

Cho HJ, Ki CS and Kim JW (2009). Improved detection of germline mutations in Korean VHL patients by multiple ligation-dependent probe amplification analysis. J. Korean Med. Sci. 24: 77-83.

Cushman RA, Tait RG, McNeel AK, Forbes ED, et al. (2015). A polymorphism in myostatin influences puberty but not fertility in beef heifers, whereas micro-calpain affects first calf birth weight. J. Anim. Sci. 93: 117-126.

Dutt P, Croall DE, Arthur JS, Veyra TD, et al. (2006). m-Calpain is required for preimplantation embryonic development in mice. BMC Dev. Biol. 6: 3 .

Equils O, Moffatt-Blue C, Ishikawa TO, Simmons CF, et al. (2009). Pretreatment with pancaspase inhibitor (Z-VADFMK) delays but does not prevent intraperitoneal heat-killed group B Streptococcus-induced preterm delivery in a pregnant mouse model. Infect Dis. Obstet. Gynecol. 749432.

Goll DE, Thompson VF, Li H, Wei W, et al. (2003). The calpain system. Physiol Rev. 83: 731-801.

Guo LF, Wang ZH and Wang YF (2016). Common variant rs7579169 is associated with preeclampsia in Han Chinese women. Genet. Mol. Res. 15: gmr8114.

Gu Y, Lewis DF, Alexander JS and Wang Y (2017). Upregulation of cathepsin C expression contributes to endothelial chymase activation in preeclampsia. Hypertens Res. 40: 976-981.

Kumagai K, Ozaki Y, Nakanishi T, Inomata M, et al. (2008). Role of mu-calpain in human decidua for recurrent miscarriage. Am. J. Reprod. Immunol. 59: 339-346.

Lee JY, Lee WJ, Hur SE, Lee CM, et al. (2009). 111/121 diplotype of Calpain-10 is associated with the risk of polycystic ovary syndrome in Korean women. Fertil Steril. 92: 830-833.

Nagai A, Sado T, Naruse K, et al. (2012). Antiangiogenic-induced hypertension: the molecular basis of signaling network. Gynecol. Obstet. Invest. 73: 89-98.

Saido TC, Sorimachi H and Suzuki K (1994). Calpain: new perspectives in molecular diversity and physiologicalpathological involvement. FASEB J. 8: 814-822.

Shinya K, Nakayama T, Nakayama T and Yamamoto T (2018). A Case-control Study between the STIM1 Gene and Hypertensive Disorders of Pregnancy. Hypertens. Res. 41: 39-44.

Shu JT, Zhang M, Shan YJ, Xu WJ, et al. (2015). Analysis of the genetic effects of CAPN1 gene polymorphisms on chicken meat tenderness. Genet. Mol. Res. 14: 1393-1403.

Tamura M, Nakayama T, Sato I, Sato N, et al. (2008). Haplotype-based case-control study of estrogen receptor $\alpha$ (ESR1) gene and pregnancy-induced hypertension. Hypertens. Res. 31: 221-228.

Takano J, Mihira N, Fujioka R, Hosoki E, et al. (2011). Vital role of the calpain-calpastatin system for placentalintegrity-dependent embryonic survival. Mol. Cell Biol. 31: 4097-4106.

Wang Y, Xiang K, Zheng T, Jia W, et al. (2002). [The UCSNP44 variation of calpain 10 gene on NIDDM1 locus and its impact on plasma glucose levels in type 2 diabetic patients]. Zhonghua Yi Xue Za Zhi. 82: 613-616.

Wu B, Takahashi J, Fu M, Cheng H, et al. (2005). Variants of calpain-10 gene and its association with type 2 diabetes mellitus in a Chinese population. Diabetes Res. Clin. Pract. 68: 155-161.

Xiang K, Fang Q, Zheng T, Jia W, et al. (2001). [The impact of calpain-10 gene combined-SNP variation on type 2 diabetes mellitus and its related metabolic traits]. Zhonghua Yi Xue Za Zhi. 18: 426-430.

Yamada M, Yoshida Y, Mori D, Takitoh T, et al. (2009). Inhibition of calpain increases LIS1 expression and partially rescues in vivo phenotypes in a mouse model of lissencephaly. Nat Med. 15: 1202-1207.

Zatz M and Starling A (2005): Calpains and disease. N. Engl. J. Med. 352: 2413-2423. 